\title{
Prognosis of Adjuvant SOX vs XELOX Chemotherapy for Gastric Cancer After D2 Gastrectomy in Chinese Patients
}

This article was published in the following Dove Press journal: Cancer Management and Research

\author{
Shan $\mathrm{Yu}^{\prime}$ \\ Yan Wang' \\ Xi Cheng $\left(\mathbb{D}^{2}\right.$ \\ Minzhi Lv ${ }^{3}$ \\ Yuehong Cui ${ }^{1}$ \\ Wei $\mathrm{Li}^{1}$ \\ Yiyi Yu (D' \\ Qian Li' \\ Tianshu Liu (D) ${ }^{1,4}$ \\ 'Department of Medical Oncology, \\ Zhongshan Hospital, Fudan University, \\ Shanghai, People's Republic of China; \\ ${ }^{2}$ Department of Medical Oncology, Sir \\ Run Run Shaw Hospital, Zhejiang \\ University, Hangzhou, People's Republic \\ of China; ${ }^{3}$ Department of Biostatistics, \\ Zhongshan Hospital, Fudan University, \\ Shanghai, People's Republic of China; \\ ${ }^{4}$ Center of Evidence-Based Medicine, \\ Fudan University, Shanghai, People's \\ Republic of China
}

Correspondence: Tianshu Liu Department of Medical Oncology, Center of Evidence-Based Medicine, Zhongshan Hospital, Fudan University, I 80 Fenglin Road, Shanghai 200032, People's Republic of China

Email liu.tianshu@zs-hospital.sh.cn
Introduction: To compare the prognosis of adjuvant SOX (S-1 and oxaliplatin) vs XELOX (capecitabine and oxaliplatin) chemotherapy in Chinese patients with gastric cancer (GC) after D2 gastrectomy.

Methods: This was a real-world study of patients with GC (stages II-III) who underwent D2 gastrectomy and received adjuvant SOX or XELOX between 01/2010 and 06/2017 in Zhongshan Hospital affiliated to Fudan University. The patients were matched by propensity score matching. The primary and secondary endpoints were disease-free survival (DFS) and overall survival (OS), respectively. Adverse events (AEs) were compared.

Results: A total of 552 patients were included. The median follow-up time was 24.9 months. There were no differences in DFS (median, 44.4 vs 41.2 months; HR=1.17, 95\% CI: $0.92-1.48$ ) and OS (median, 61.5 vs 65.3 months; HR=1.01, 95\% CI: $0.73-1.39$ ) between the XELOX and SOX groups. Both DFS and OS had no significant differences between SOX and XELOX for all subgroups based on sex $(\mathrm{P}=0.949, \mathrm{P}=0.990)$, age $(\mathrm{P}=0.303, \mathrm{P}=0.392)$, Lauren type $(\mathrm{P}=0.362, \mathrm{P}=0.573)$, type of gastrectomy $(\mathrm{P}=0.607 \mathrm{P}=0.989)$, and pathological TNM stage $(\mathrm{P}=0.899, \mathrm{P}=0.888)$. A total of 86 patients in the SOX subgroup $(34.2 \%)$ experienced AEs, similar to the rate found in the XELOX subgroup (104 patients or 41.4\%; $\mathrm{P}=0.098$ ).

Discussion: The results suggested that adjuvant SOX chemotherapy has similar survival benefits compared to XELOX chemotherapy in Chinese patients with pathological stage II or III GC after D2 gastrectomy.

Keywords: gastric cancer, gastrectomy, adjuvant chemotherapy, capecitabine, oxaliplatin, S-1, XELOX

\section{Introduction}

Gastric cancer (GC) is one of the most common malignancies in the world, with an annual incidence of about one million, making it the fifth most common cancer and the third leading cause of cancer-related deaths worldwide. ${ }^{1,2}$ The highest rates of estimated morbidity and mortality have been observed in Eastern Asian countries. ${ }^{1}$

Although radical gastrectomy with D2 lymphadenectomy is considered the most beneficial curative option, ${ }^{3}$ the chances of tumor recurrence and metastasis still constitute major concerns and drawbacks after surgery in Eastern countries ${ }^{4-6}$ Fortunately, a large number of clinical trials have shown that adjuvant chemotherapy decreases the risk of recurrence and improves the survival of patients with 
GC. ${ }^{7-11}$ Nevertheless, the most optimal regimen has not been clearly identified for adjuvant chemotherapy after D2 gastrectomy.

The multicenter CLASSIC trial in Eastern Asia showed that adjuvant oxaliplatin plus capecitabine (XELOX regimen) after curative D2 gastrectomy improves disease-free survival (DFS) and overall survival (OS) compared with surgery alone. ${ }^{7,8}$ Another trial showed that S-1 (an oral combination of tegafur, gimeracil, and oteracil at a molar ratio of 1:0.4:1) improves survival after D2 gastrectomy in Japan. ${ }^{12}$ Both capecitabine and S-1, which are oral fluoropyrimidines, have been proposed as substitutes for continuous infusion of 5-FU because they are more convenient and have lower risks. ${ }^{13,14}$ The Japanese ACTS-GC trial showed that adjuvant S-1 monotherapy could improve relapse-free survival (RFS) and OS rates. ${ }^{12}$ As it is hard to complete a 1-year course of adjuvant S-1 monotherapy, it is difficult to achieve satisfactory clinical expectations; therefore, it is essential to investigate the efficacy of S-1 combined with oxaliplatin as adjuvant treatment for GC.

Recently, adjuvant oxaliplatin plus S-1 (SOX regimen) for GC has been shown to be associated with survival benefits, and adjuvant SOX is considered an effective treatment alternative for GC. ${ }^{9-11}$ A previous study by our group suggested that oxaliplatin-based adjuvant chemotherapy regimens (such as XELOX and SOX) show remarkable survival benefits in patients with intestinal-type GC after D2 gastrectomy. ${ }^{15}$ A Phase III trial of XELOX vs SOX after D2 gastrectomy suggested that both regimens could achieve similar DFS. ${ }^{16}$ Another study examined the efficacy of adjuvant SOX vs XELOX after D2 gastrectomy for stage III GC, and showed that both regimens achieved similar benefits, but highlighted that additional data are needed in subgroups of patients, especially regarding sex and histological type. ${ }^{17}$

Therefore, the aim of this study was to compare the prognosis of patients who received adjuvant SOX vs XELOX in GC.

\section{Materials and Methods Study Design and Patients}

This was a real-world study using a database of patients with GC (stages I to IV), who underwent D2 gastrectomy and received adjuvant chemotherapy between January 2010 and June 2017 in Zhongshan Hospital affiliated to Fudan University. This study was approved by the Ethics Committee of Zhongshan Hospital affiliated to Fudan University. The need for individual consent was waived by the committee, because this real-world study retrospectively retrieved data from medical records. Patient data were confidential, and the study was in compliance with the Declaration of Helsinki.

Inclusion criteria were: 1) histologically proven gastric adenocarcinoma after radical gastrectomy with D2 lymph node dissection; 2) 20-75 years of age, with adequate organ functions; 3) no neoadjuvant chemotherapy or radiotherapy; 4) pathological stage II or III GC according to the 8th edition of the AJCC cancer staging manual; 5) adjuvant XELOX or SOX; 6) no adjuvant radiotherapy or chemoradiotherapy within 6 months after surgery; and 7) no synchronous or metachronous cancer. Exclusion criteria were: 1) positive resection margin; 2) metastatic disease (M1); or 3) change of adjuvant chemotherapy regimen during treatment.

\section{Data Collection}

Sex, age, pathological and clinical TNM stage, detailed pathological information, chemotherapy regimen, diagnosis date, operation date, dates of initiation and termination of chemotherapy, recurrence or progression date, followup dates, and death date were extracted from the database. Complete blood count and blood chemistry analyses were routinely performed before the initiation of each cycle. Adverse events (AEs) were evaluated using the National Cancer Institute-Common Toxicity Criteria version 3.0.

\section{Chemotherapy Regimen}

All patients received adjuvant chemotherapy within 6 weeks after surgery, according to the National Comprehensive Cancer Network $(\mathrm{NCCN})^{3}$ and the Chinese Society of Clinical Oncology $(\mathrm{CSCO})^{18}$ guidelines. Patients were administered 8 cycles of the XELOX or SOX regimen for 6 months. The XELOX regimen consisted of 3 -week cycles of oxaliplatin $\left(130 \mathrm{mg} / \mathrm{m}^{2}\right.$ on day 1 of each cycle, intravenously) plus capecitabine (1000 $\mathrm{mg} / \mathrm{m}^{2}$ twice daily on days $1-14$, orally). The SOX regimen consisted of 3 -week cycles of oxaliplatin $\left(130 \mathrm{mg} / \mathrm{m}^{2}\right.$ on day 1 of each cycle, intravenously) plus S-1 (daily oral dose of 80,100 , or $120 \mathrm{mg}$ in two separate administrations on the basis of body surface area on days 1-14). Both regimens have the same indications. ${ }^{18}$

Adverse events were graded according to the National Cancer Institute's Common Terminology Criteria for Adverse Events (version 3.0). All patients were assessed for adverse events during chemotherapy and within 28 days after the last dose of medication. 


\section{Follow-Up}

Patients were followed for DFS and OS every 3 months within 2 years after surgery, every 6 months within 5 years, and every 12 months beyond 5 years. All patients were followed routinely by physical examination, serum tumor marker evaluation, chest and abdominal computed tomography (CT), and gastrointestinal endoscopic examination. As per routine practice, whole-body bone scan, abdominal magnetic resonance imaging (MRI), and positron emission tomography/CT (PET/CT) scan were performed if needed.

\section{Endpoints}

The dates of first relapse and death were recorded. The primary and secondary endpoints were DFS and OS, respectively. DFS was determined from the date of operation to the date of recurrence or metastasis, new GC, or death from any cause. OS was defined as the period from the date of operation to last follow-up or death for any reason.

\section{Statistical Analysis}

Age was dichotomized. Categorical data were presented as numbers and percentages, and analyzed by the chi-square test. Continuous data were tested for normality by the Kolmogorov-Smirnov test, presented as means \pm standard deviations, and analyzed by the Student's $t$-test. DFS and OS were estimated by the Kaplan-Meier method. Propensity score matching (PSM) accounted for age, sex, Lauren type, type of gastrectomy, pathological tumor $(\mathrm{T})$ stage, and pathological node $(\mathrm{N})$ stage, and was performed using a logistic regression model and the nearest neighbor matching algorithm with a ratio of $1: 1$. A difference of $<10 \%$ of the absolute value was considered to be balanced. Estimates of treatment benefits were calculated as hazard ratios (HRs) with $95 \%$ confidence intervals (CIs). Significant variables in univariable analysis were further investigated by a multivariable Cox proportional hazards model. A generalized linear model (GLM) adjusted for age, sex, Lauren classification, type of gastrectomy, and pathological TNM stage was used to compare survival between the two groups. Analyses were performed with SPSS 23.0 (IBM, Armonk, NY, USA). P-values $<0.05$ were considered statistically significant.

\section{Results}

\section{Characteristics of the Patients}

From January 2010 to June 2017, a total of 552 patients satisfied all the eligibility criteria, and 517 were excluded from the study (Figure 1). There were 118 patients (21.4\%) with pathological stage II and 434 (78.6\%) with pathological stage III GC. According to the Lauren classification, there were 164 patients $(29.7 \%)$ with intestinaltype GC, $232(42.0 \%)$ with diffuse-type GC, and 156 $(28.3 \%)$ with mixed-type GC. Among the 552 patients, $251(45.5 \%)$ received adjuvant SOX, and 301 (54.5\%) were administered adjuvant XELOX.

The baseline characteristics of the patients before and after PSM are shown in Table 1. After adjustment of background factors by PSM, both groups were well balanced with respect to sex, age, Lauren type, type of gastrectomy, pathological $\mathrm{T}$ stage, and pathological $\mathrm{N}$ stage (all $\mathrm{P}>0.05$ ).

\section{Follow-Up}

The last follow-up was performed in June 2018, and the median follow-up time was 24.9 (range, 3.2-103.2) months. Of the 552 patients, 305 (55.4\%; 130 and 175 in the SOX and XELOX groups, respectively) showed metastasis or recurrence, and 170 died $(30.8 \%$; 64 and 106 in the SOX and XELOX groups, respectively) by the last followup day.

Before PSM, there were no significant differences between patients receiving SOX and XELOX for median DFS (HR $=1.11,95 \%$ CI: 0.89-1.41) and median OS (HR $=0.97,95 \%$ CI: 0.71-1.33). After PSM, there were no differences in DFS (median, 44.4 vs 41.2 months; HR $=1.17$, 95\% CI: $0.92-1.48$ ) and OS (median, 61.5 vs 65.3 months; HR=1.01, 95\% CI: $0.73-$ 1.39) between the XELOX and SOX groups (Table 2). Similar results were observed using the GLM (both 95\% CIs crossing 1).

After PSM, there were no significant differences in median DFS (SOX 24.8 vs XELOX 28.7 months $\mathrm{P}=0.196 ; \mathrm{HR}=1.17,95 \% \mathrm{CI}:$ 0.92-1.48) (Figure 2A) and median OS (SOX 69.8 vs XELOX 59.4 months, $\mathrm{P}=0.966 ; \mathrm{HR}=1.01,95 \% \mathrm{CI}:$ 0.71-1.39) (Figure 2B) between the two chemotherapy regimens. Among patients with pathological stage II GC, SOX did not lead to significantly longer DFS (SOX 50.5 vs XELOX 57.5, $\mathrm{P}=0.891 ; \mathrm{HR}=1.05,95 \% \mathrm{CI}: 0.55-2.00$ ) (Figure $2 \mathrm{C}$ ) or OS (SOX not reached vs XELOX not reached, $\mathrm{P}=0.852 ; \mathrm{HR}=1.11,95 \% \mathrm{CI}: 0.38-3.22$ ) (Figure 2D) compared with the XELOX subgroup. In patients with pathological stage III GC, similar findings were obtained, and DFS (SOX 20.2 vs XELOX 26.2 months, $\mathrm{P}=0.182 ; \mathrm{HR}=1.19,95 \% \mathrm{CI}: 0.92-1.54$ ) (Figure 2C) and 


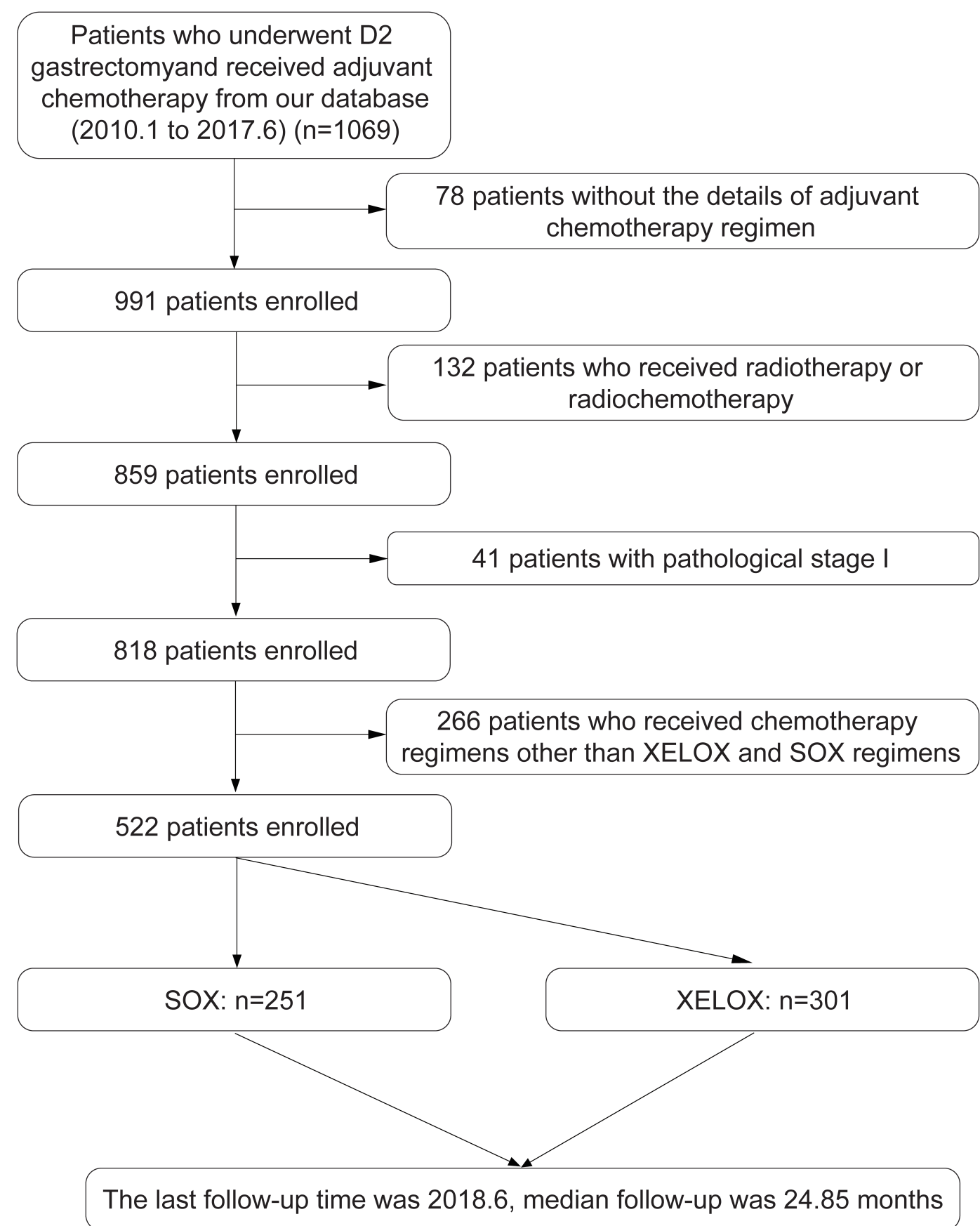

Figure I Patient flowchart. According to the eligibility criteria, 517 were excluded from the 1069 patients identified, and 552 patients were analyzed.

OS (SOX 69.8 vs XELOX 52.0 months, $\mathrm{P}=0.976$; $\mathrm{HR}=0.99$, 95\% CI: 0.71-1.39) (Figure 2D) were not significantly different between the SOX and XELOX regimens. In patients with pathological stage III GC, Kaplan-Meier curves for DFS or OS did not show differences between the two adjuvant regimens for the intestinal (DFS, $\mathrm{HR}=1.07$, 95\% CI: 0.61-1.89; OS, $\mathrm{HR}=1.00,95 \% \mathrm{CI}: 0.47-2.13$ ), diffuse (DFS, $\mathrm{HR}=1.05$,
95\% CI: $0.73-1.51$; OS, HR=0.93, 95\% CI: $0.58-1.48$ ) and mixed (DFS, HR=1.57, 95\% CI: 0.99-2.57; OS, $\mathrm{HR}=1.10,95 \% \mathrm{CI}: 0.60-2.05$ ) types (Figure 2E and F).

\section{Subgroup Analyses}

Figure 3 shows that DFS was similar between the SOX and XELOX regimens for all subgroups based on sex ( $\mathrm{P}=0.949)$, age $(\mathrm{P}=0.303)$, Lauren type $(\mathrm{P}=0.362)$, type 
Table I Characteristics of the Patients Administered SOX or XELOX

\begin{tabular}{|c|c|c|c|c|c|c|}
\hline \multirow[t]{2}{*}{ Characteristics } & \multicolumn{3}{|l|}{ Before PSM } & \multicolumn{3}{|l|}{ After PSM } \\
\hline & $\begin{array}{l}\text { SoX Subgroup } \\
(n=25 I)\end{array}$ & $\begin{array}{l}\text { XELOX Subgroup } \\
(n=301)\end{array}$ & $\mathbf{P}$ & $\begin{array}{l}\text { soX Subgroup } \\
(n=25 I)\end{array}$ & $\begin{array}{l}\text { XELOX Subgroup } \\
(n=25 I)\end{array}$ & $\mathbf{P}$ \\
\hline $\begin{array}{l}\text { Sex } \\
\qquad \text { Male } \\
\text { Female }\end{array}$ & $\begin{array}{l}163(64.9 \%) \\
88(35.5 \%)\end{array}$ & $\begin{array}{l}210(69.8 \%) \\
91(30.2 \%)\end{array}$ & 0.228 & $\begin{array}{l}163(64.9 \%) \\
88(35.5 \%)\end{array}$ & $\begin{array}{l}174(69.3 \%) \\
77(30.7 \%)\end{array}$ & 0.296 \\
\hline $\begin{array}{l}\text { Age (years) } \\
\quad>60 \\
\quad \leq 60\end{array}$ & $\begin{array}{l}96(38.2 \%) \\
155(61.8 \%)\end{array}$ & $\begin{array}{l}132(43.8 \%) \\
169(56.1 \%)\end{array}$ & 0.183 & $\begin{array}{l}96(38.2 \%) \\
155(61.8 \%)\end{array}$ & $\begin{array}{l}116(46.2 \%) \\
135(53.8 \%)\end{array}$ & 0.071 \\
\hline $\begin{array}{l}\text { Lauren type } \\
\text { Intestinal } \\
\text { Diffuse } \\
\text { Mixed }\end{array}$ & $\begin{array}{l}68(27.1 \%) \\
105(41.8 \%) \\
78(31.1 \%)\end{array}$ & $\begin{array}{l}96(31.9 \%) \\
127(42.2 \%) \\
78(25.9 \%)\end{array}$ & 0.308 & $\begin{array}{l}68(27.1 \%) \\
105(41.8 \%) \\
78(31.1 \%)\end{array}$ & $\begin{array}{l}80(31.9 \%) \\
99(39.4 \%) \\
72(28.7 \%)\end{array}$ & 0.499 \\
\hline $\begin{array}{l}\text { Type of gastrectomy } \\
\text { Total } \\
\text { Distal } \\
\text { Proximal }\end{array}$ & $\begin{array}{l}\text { II } 9 \text { (47.4\%) } \\
\text { II (47.0\%) } \\
\text { I4 (5.6\%) }\end{array}$ & $\begin{array}{l}\text { I } 26(41.9 \%) \\
\text { I59 (52.8\%) } \\
\text { I6 (5.3\%) }\end{array}$ & 0.389 & $\begin{array}{l}119(47.4 \%) \\
118(47.0 \%) \\
14(5.6 \%)\end{array}$ & $\begin{array}{l}102(40.6 \%) \\
136(54.2 \%) \\
13(5.2 \%)\end{array}$ & 0.270 \\
\hline $\begin{array}{l}\text { Pathological T-stage } \\
\text { TI } \\
\text { T2 } \\
\text { T3 } \\
\text { T4 }\end{array}$ & $\begin{array}{l}5(2.0 \%) \\
25(10.0 \%) \\
69(27.5 \%) \\
152(60.5 \%)\end{array}$ & $\begin{array}{l}8(2.7 \%) \\
35(11.6 \%) \\
57(18.9 \%) \\
201(66.8 \%)\end{array}$ & 0.121 & $\begin{array}{l}5(2.0 \%) \\
25(10.0 \%) \\
69(27.5 \%) \\
152(60.5 \%)\end{array}$ & $\begin{array}{l}5(2.0 \%) \\
26(10.4 \%) \\
44(17.5 \%) \\
176(70.1 \%)\end{array}$ & 0.063 \\
\hline $\begin{array}{l}\text { Pathological N-stage } \\
\text { N0 } \\
\text { NI } \\
\text { N2 } \\
\text { N3 }\end{array}$ & $\begin{array}{l}21(8.4 \%) \\
35(13.9 \%) \\
75(29.8 \%) \\
120(47.9 \%)\end{array}$ & $\begin{array}{l}29(9.6 \%) \\
40(13.3 \%) \\
76(25.2 \%) \\
156(51.9 \%)\end{array}$ & 0.615 & $\begin{array}{l}21(8.4 \%) \\
35(13.9 \%) \\
75(29.8 \%) \\
120(47.9 \%)\end{array}$ & $\begin{array}{l}21(8.4 \%) \\
33(13.1 \%) \\
68(27.1 \%) \\
129(51.4 \%)\end{array}$ & 0.867 \\
\hline $\begin{array}{l}\text { Pathological TNM } \\
\text { stage } \\
\text { II } \\
\text { III }\end{array}$ & $\begin{array}{l}50(19.9 \%) \\
20 I(80.1 \%)\end{array}$ & $\begin{array}{l}68(22.6 \%) \\
233(77.4 \%)\end{array}$ & 0.446 & $\begin{array}{l}50(19.9 \%) \\
201(80.1 \%)\end{array}$ & $\begin{array}{l}53(21.1 \%) \\
198(78.9 \%)\end{array}$ & 0.740 \\
\hline
\end{tabular}

Abbreviations: PSM, propensity score matching; SOX, S-I and oxaliplatin; XELOX, oxaliplatin and capecitabine; TNM, tumor node metastasis.

of gastrectomy ( $\mathrm{P}=0.607)$, and pathological TNM stage subgroups based on sex $(\mathrm{P}=0.990)$, age $(\mathrm{P}=0.392)$, $(\mathrm{P}=0.899)$. Figure 4 shows that $\mathrm{OS}$ was also similar Lauren type $(\mathrm{P}=0.573)$, type of gastrectomy $(\mathrm{P}=0.989)$, between the SOX and XELOX regimens for all and pathological TNM stage $(\mathrm{P}=0.888)$.

Table 2 DFS and OS of the XELOX and SOX Subgroups After PSM or GLM

\begin{tabular}{|l|l|l|l|l|l|l|}
\hline \multicolumn{2}{|l|}{ DFS (Months) } & \multicolumn{2}{l|}{ OS (Months) } & HR (95\% CI) \\
\cline { 2 - 7 } & XELOX & SOX & HR (95\% Cl) & XELOX & SOX & $1.01(0.73-1.39)$ \\
\hline PSM & 44.4 & 41.2 & $1.17(0.92-1.48)$ & 61.5 & 65.3 & $0.91(0.66-1.25)$ \\
GLM* & 41.8 & 41.2 & $1.06(0.84-1.34)$ & 60.5 & 65.3 & \\
\hline
\end{tabular}

Note: *Adjusted for age, sex, Lauren classification, type of gastrectomy, and pathological TNM stage.

Abbreviations: DFS, disease-free survival; OS, overall survival; SOX, S-I and oxaliplatin; XELOX, oxaliplatin and capecitabine; HR, hazards ratio; Cl, confidence interval; PSM, propensity-matched analysis; GLM, generalized linear model. 
A

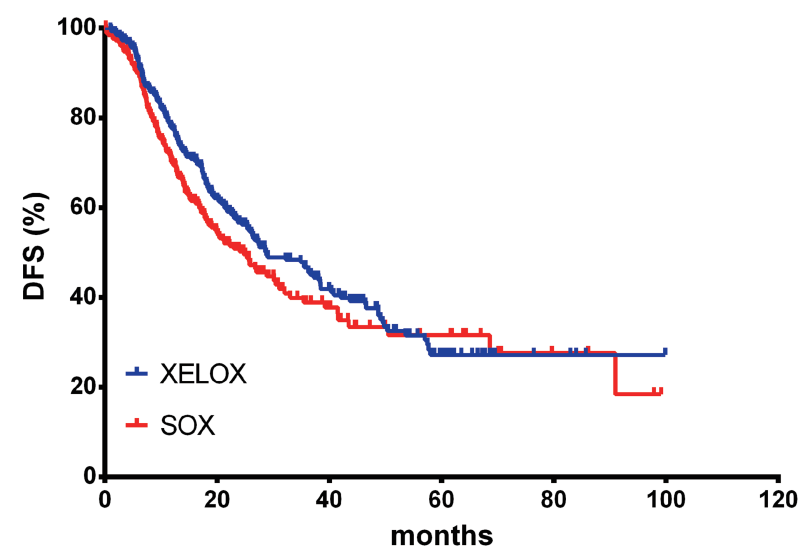

C

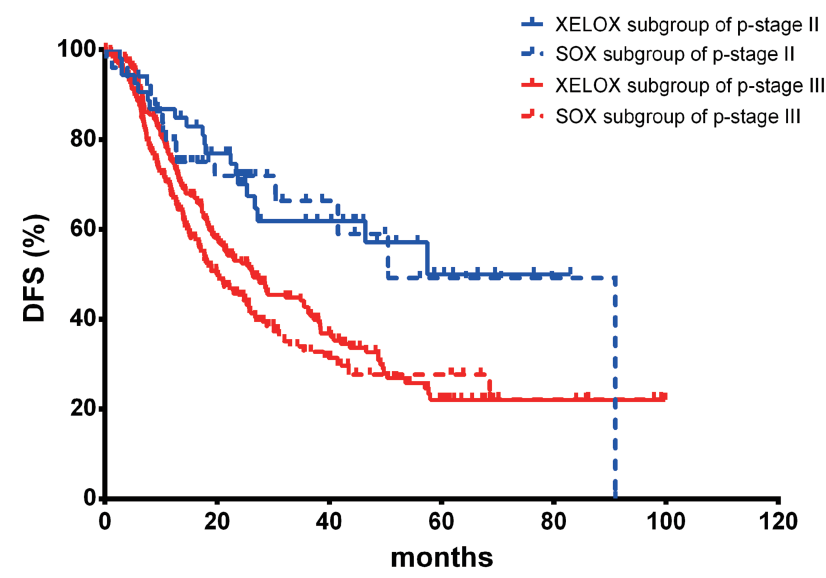

E

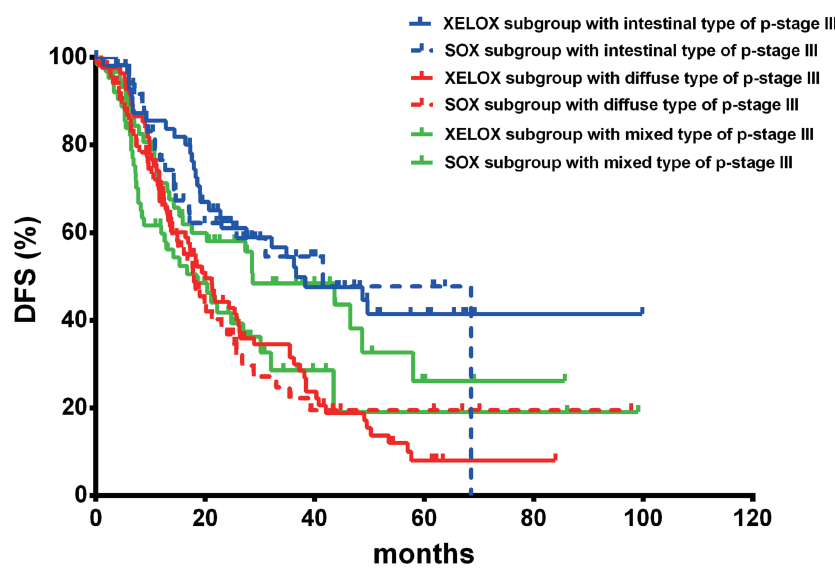

B

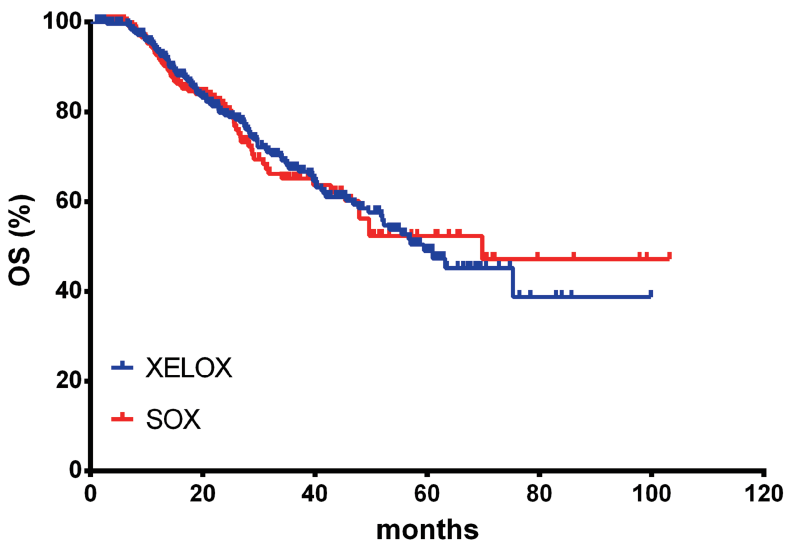

D

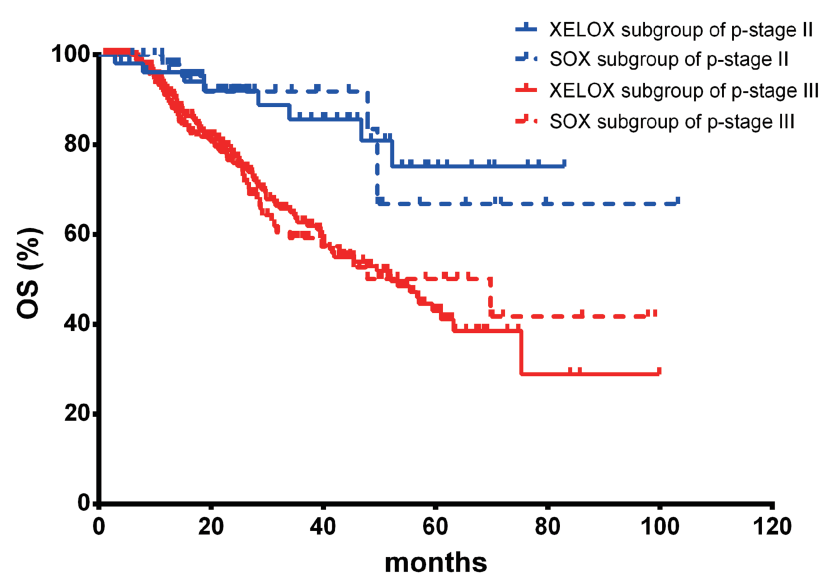

F

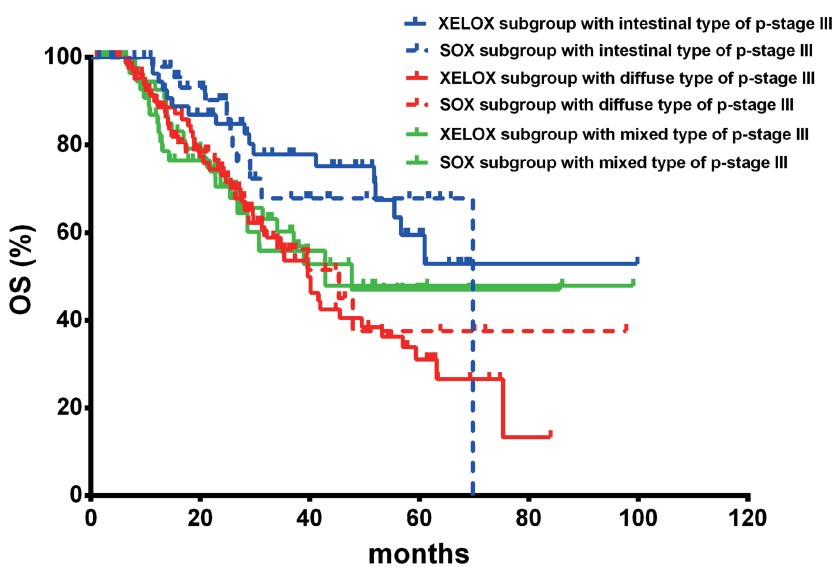

Figure 2 Kaplan-Meier survival curves for disease-free survival (DFS) and overall survival (OS) after propensity score matching (PSM). DFS (A) and OS (B) analyses for the XELOX and SOX regimens. DFS (C) and OS (D) analyses of patients with pathological stage II and III GC treated with the SOX and XELOX regimens. DFS (E) and OS (F) analyses of patients treated with the SOX and XELOX regimens in different Lauren type GC of pathological stage III disease.

\section{Adverse Events}

AEs in the SOX and XELOX groups after PSM are shown in Table 3. A total of 86 patients in the SOX group
(34.2\%) experienced AEs, similar to the rate found in the XELOX subgroup (104 patients or 41.4\%; $\mathrm{P}=0.098$ ). There were no differences in nausea/vomiting, 


\section{Subgroup Analyses of Disease Free Survival}

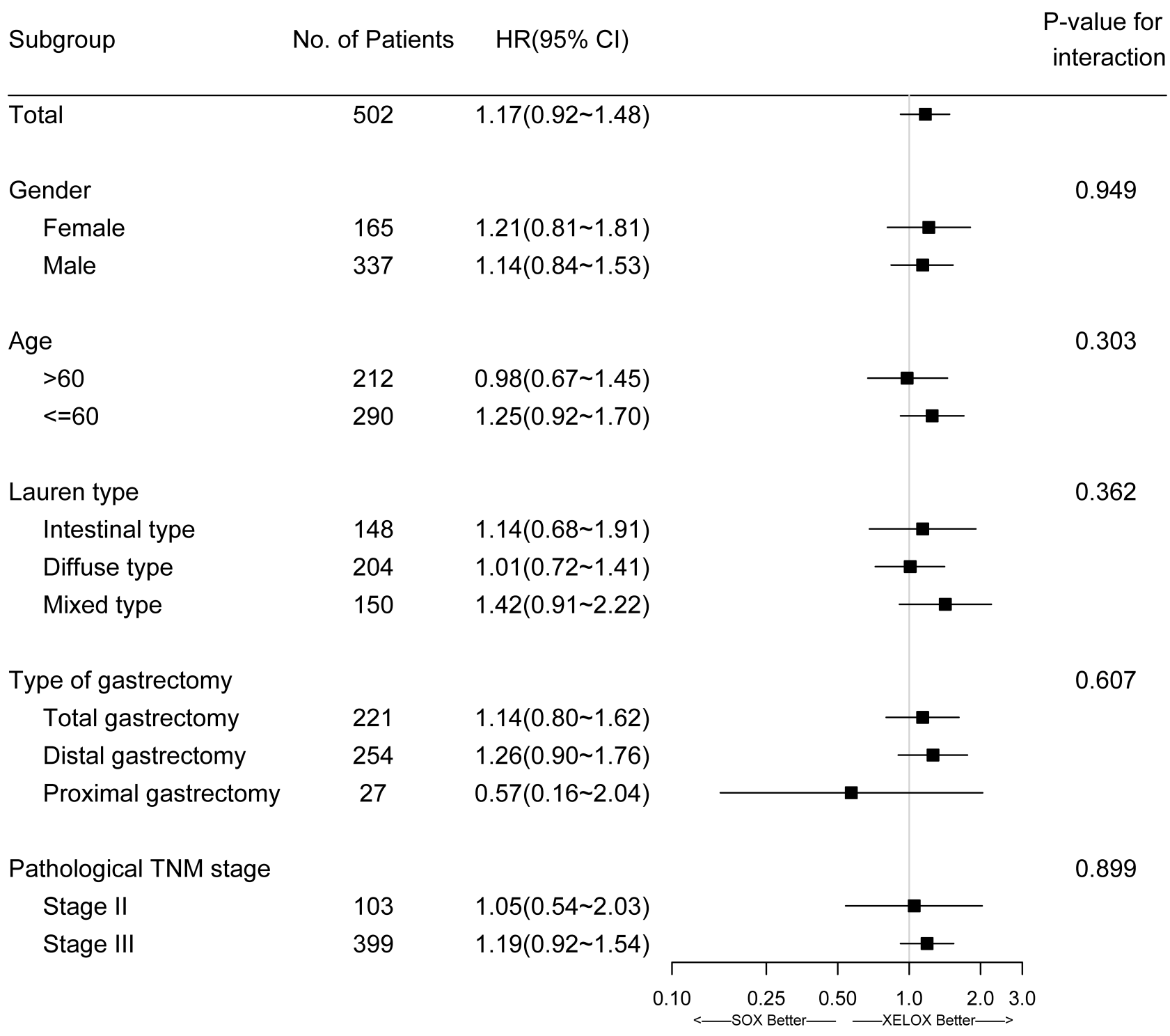

Figure 3 Subgroup analyses of disease-free survival (DFS) after propensity score matching (PSM).

myelosuppression, peripheral neurotoxicity, and hand-foot syndrome (all $\mathrm{P}>0.05$ ). The most common adverse events were gastrointestinal issues and myelosuppression.

\section{Discussion}

A previous study suggested that SOX and XELOX achieve similar survival in patients with GC after gastrectomy, ${ }^{17}$ but additional data in subgroups were needed. Therefore, this study aimed to compare adjuvant SOX (S-1 and oxaliplatin) and XELOX (capecitabine and oxaliplatin) chemotherapy for survival benefits in Chinese patients with gastric cancer (GC) after D2 gastrectomy. The results suggested that adjuvant SOX chemotherapy resulted in similar survival benefits compared to XELOX chemotherapy in Chinese patients with pathological stage II or III GC after D2 gastrectomy. The AE profiles were also similar between the two regimens.

In Eastern countries, the most widely used therapeutic procedure for the treatment of GC is radical gastrectomy with D2 lymph node dissection, ${ }^{19,20}$ and this procedure is gaining popularity in Western countries. ${ }^{19}$ Nevertheless, chemotherapy after gastrectomy is required. ${ }^{3}$ The CLASSIC trial performed in 37 centers in South Korea, China, and Taiwan showed significantly higher rates of 


\section{Subgroup Analyses of Overall Survival}

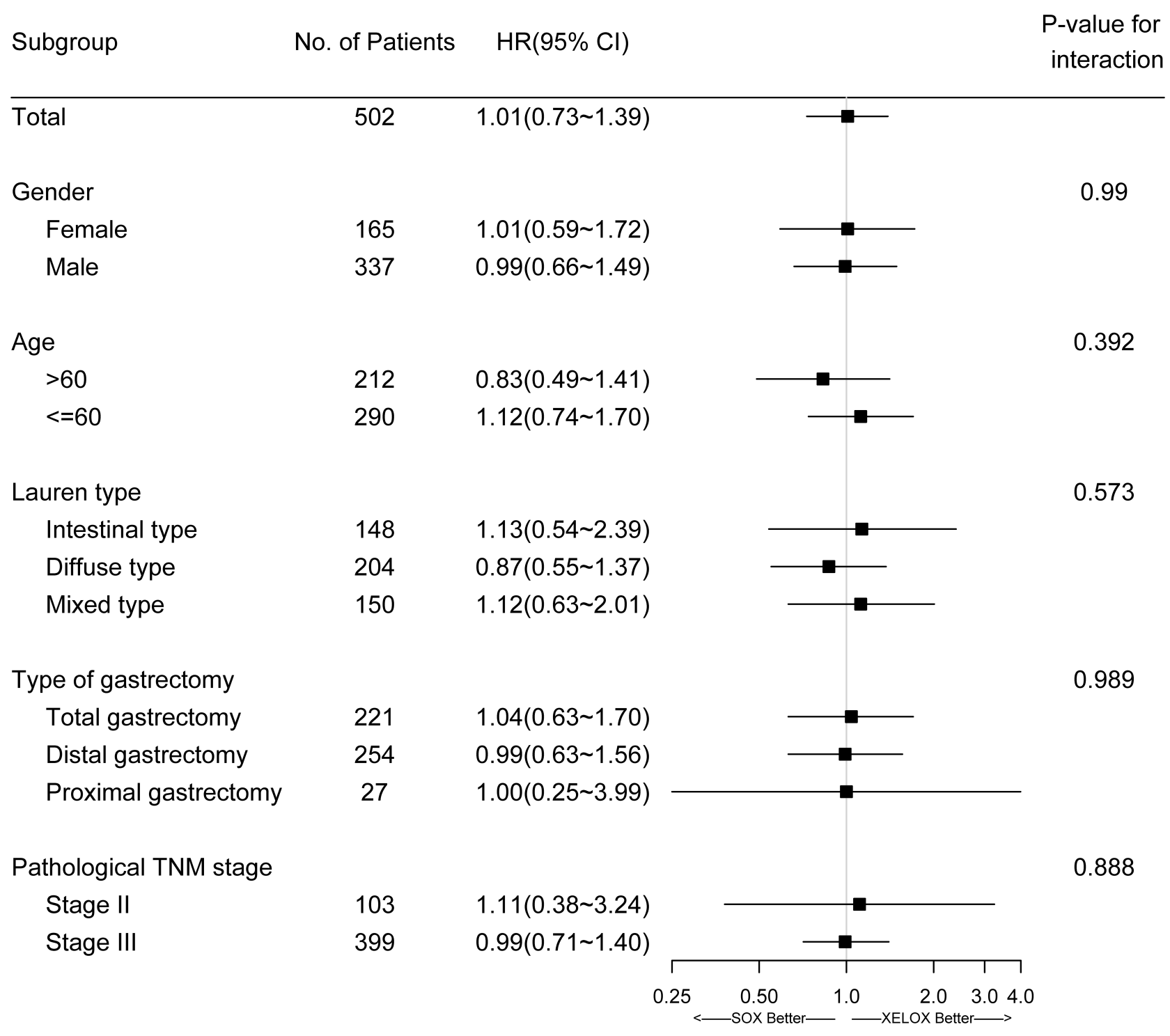

Figure 4 Subgroup analyses of overall survival (OS) after propensity score matching (PSM).

DFS and OS in the adjuvant XELOX group compared with the surgery alone group. ${ }^{7,8}$ SOX is an emerging chemotherapy regimen widely used in China and Japan. ${ }^{10,11,17,21-23}$ A randomized phase III trial of metastatic cases suggested that SOX is not less potent than CS (cisplatin plus S-1) in terms of PFS and OS. ${ }^{23}$ Compared with the CS regimen, the SOX regimen has several benefits, including no need for hydration due to low renal toxicity. ${ }^{23}$ In 2017, a Phase II trial revealed that SOX chemotherapy is manageable and safe for pathological stage III GC after D2 gastrectomy. ${ }^{9}$ Another phase II trial of SOX in China showed 1- and 3-year DFS rates of $85.2 \%$ and $75.9 \%$, respectively, while 1 - and 3 -year OS rates were $98.1 \%$ and $85.2 \%$, respectively. ${ }^{10}$ The results of the RESOLVE trial were announced at the ESMO Annual Meeting in 2019. ${ }^{16}$ This was a phase III clinical study initiated in China and carried out in pathologically confirmed patients with adenocarcinoma of the stomach or gastroesophageal junction. The participants were randomized to 8 cycles of adjuvant XELOX, 8 cycles of adjuvant SOX, and 3 cycles of neoadjuvant SOX. The 3-year DFS rates were $54.8 \%, 60.3 \%$, and $62.0 \%$, respectively, without significant differences. Hence, the RESOLVE trial concluded that SOX was not inferior to XELOX in 3-year 


\begin{tabular}{|l|l|l|l|}
\hline Event & SOX Subgroup (n=25I) & XELOX Subgroup (n=25I) & P \\
\hline At least one adverse event & $86(34.2 \%)$ & $104(41.4 \%)$ & 0.098 \\
Nausea/vomiting & $42(16.7 \%)$ & $40(15.9 \%)$ & 0.809 \\
Myelosuppression & $41(18.7 \%)$ & $52(20.7 \%)$ & 0.206 \\
Peripheral neurotoxicity & $22(8.8 \%)$ & $35(13.9 \%)$ & 0.067 \\
Hand-foot syndrome & $5(2.0 \%)$ & $3(1.2 \%)$ & 0.724 \\
\hline
\end{tabular}
present study, which was carried out in a real-world setting.

In Japan, a study by Nakamura et al ${ }^{17}$ suggested that SOX and XELOX could achieve similar survival in patient with GC after D2 gastrectomy, but highlighted that additional subgroup data are needed. In China, Cheng et al ${ }^{15}$ showed that oxaliplatin-based adjuvant chemotherapy regmens (such as XELOX and SOX) have substantial surviva benefits in patients with intestinal-type GC after D2 gastrectomy. Recently, the JACCRO GC-07 trial demonstrated a better survival after treatment with docetaxel plus S-1 compared with S-1 monotherapy after D2 gastrectomy fo Stage III GC. ${ }^{24}$ As a control for docetaxel + S-1 the latter trial assessed S-1 monotherapy, rather than the SOX XELOX regimen. Currently, there is no reported head-tohead comparison between the docetaxol+S-1 and SOX XELOX regimens. In addition, no randomized phase II studies have confirmed that postoperative S1 monotherapy in stage III gastric cancer is not inferior to the SOX/XELOX regimen. The present study suggested that DFS and OS did not differ significantly between adjuvant SOX and XELOX chemotherapies in Chinese patients with pathological stage II and III GC after D2 gastrectomy.

At the 2018 ASCO meeting, a survival analysis of two phase II trials conducted in Japan reported HRs for SOX vs

XELOX of 0.93 ( $95 \%$ CI: $0.50-1.72, \mathrm{P}=0.81)$ for RFS an 1.10 (95\% CI: $0.54-2.26, \mathrm{P}=0.79)$ for OS in patients with pathological stage III GC. Furthermore, Ren et $\mathrm{al}^{22}$ showed that in patients with pathological stage IB to IIIC GC after D2 dissection, SOX has no significant differences in DFS and OS compared to the XELOX regimen after PSM. In the latter study, estimated 5-year DFS and OS were similar in the adjuvant SOX and XELOX groups (DFS, HR $=0.658$ $95 \% \quad \mathrm{Cl}: \quad 0.360-1.203 ; \quad \mathrm{OS}, \quad \mathrm{HR}=0.714, \quad 95 \% \quad \mathrm{Cl}$ $0.382-1.334){ }^{22}$ Those findings were consistent with randomized trials showing similar survival outcomes for SOX in advanced $\mathrm{GC}^{21,25,26} \mathrm{~A}$ randomized phase II trial reported that the SOX and XELOX regimens are equally effective in patients with advanced $\mathrm{GC}^{21} \mathrm{~A}$ previous study by our group showed that oxaliplatin-based adjuvant chemotherapy regimens are associated with improved survival rate in intestinal-type GC, while these effects were not observed in diffuse-type GC after D2 gastrectomy, compared with oxaliplatin-free adjuvant chemotherapy regimens. ${ }^{15}$ In this previous study, only the intestinal and diffuse types of $\mathrm{GC}$, but not the mixed type, were assessed. Here, SOX seemed to show similar benefits in patients with intestinal-type, diffuse-type, or mixed-type GC compared to XELOX. Interestingly, a recent meta-analysis demonstrated that thirdline (TLT) and salvage (ST) treatments are both superior to placebo or best supportive care in increasing OS and progression-free survival in advanced or metastatic $\mathrm{GC},{ }^{27}$ and SOX or XELOX could be applied in such settings based on our findings.

As a strength, this was a real-world study including patients with stage II and III GC detected based on postoperative pathological evaluation. Therefore, the present findings are broadly applicable to $\mathrm{GC}$ patients. In addition, propensity score matching was used in this study to achieve better comparability between the two groups of patients.

However, the present study had some limitations because of its real-world and non-randomized design, including patients from a single center. The number of eligible patients may not be high enough, and the follow-up duration may be too short. Therefore, a large multi-center randomized study should further evaluate the prognostic effects of different adjuvant chemotherapy regimens with long-term follow-up. In addition, the present study was limited to Chinese patients with GC who received adjuvant SOX or XELOX after D2 gastrectomy. Thus, adjuvant SOX chemotherapy in other populations remains to be investigated because of potential differences in the pharmacokinetics and toxicity of S-1 between Western and Asian patients. ${ }^{28}$ Furthermore, a global study should be conducted with standardized D2 dissection. 


\section{Conclusion}

The above results suggest that adjuvant SOX chemotherapy has similar survival benefits compared to XELOX chemotherapy in Chinese patients with pathological stage II or III GC after D2 gastrectomy. The AE profiles were also similar between the two regimens.

\section{Ethics Approval and Consent to Participate}

This study was approved by the Ethics Committee of Zhongshan Hospital affiliated to Fudan University. The need for individual consent was waived by the committee, because this real-world study retrospectively retrieved data from medical records.

\section{Author Details}

Shan Yu: 0456233@fudan.edu.cn

Yan Wang: wang.yan@zs-hospital.sh.cn

Xi Cheng: chengxi0580@126.com

Minzhi Lv: lv.minzhi@zs-hospital.sh.cn

Yuehong Cui:cui.yuehong@zs-hospital.sh.cn

Wei Li: 0256194@fudan.edu.cn

Yiyi Yu: yu.yiyi@zs-hospital.sh.cn

Qian Li: limimi0303@163.com

\section{Author Contributions}

All authors made substantial contributions to conception and design, acquisition of data, or analysis and interpretation of data; took part in drafting the article or revising it critically for important intellectual content; agreed on the journal to which the article will be submitted; gave final approval of the version to be published; and agree to be accountable for all aspects of the work.

\section{Funding}

This work was supported by grants from the National Nature Science Foundation of China (Grant No. 81702965) and the Shanghai Committee of Science and Technology, China (Grant No. 17411951400).

\section{Disclosure}

None of the authors declared any conflict of interest.

\section{References}

1. Bray F, Ferlay J, Soerjomataram I, Siegel RL, Torre LA, Jemal A. Global cancer statistics 2018: GLOBOCAN estimates of incidence and mortality worldwide for 36 cancers in 185 countries. CA Cancer J Clin. 2018;68(6):394-424. doi:10.3322/caac.21492
2. Siegel RL, Miller KD, Jemal A. Cancer statistics, 2020. CA Cancer J Clin. 2020;70(1):7-30. doi:10.3322/caac.21590

3. NCCN Clinlcal Practice Guidelines in Oncology (NCCN Guidelines). Gastric Cancer. Version 4.2019. Fort Washington: National Comprehensive Cancer Network; 2019.

4. Gallo A, Cha C. Updates on esophageal and gastric cancers. World $J$ Gastroenterol. 2006;12(20):3237-3242. doi:10.3748/wjg.v12. i20.3237

5. Liu D, Lu M, Li J, et al. The patterns and timing of recurrence after curative resection for gastric cancer in China. World J Surg Oncol. 2016;14(1):305. doi:10.1186/s12957-016-1042-y

6. Wada T, Kunisaki C, Hasegawa S, et al. Factors predictive of recurrence after surgery for gastric cancer followed by adjuvant S-1 chemotherapy. Anticancer Res. 2013;33(4):1747-1751.

7. Bang YJ, Kim YW, Yang HK, et al. Adjuvant capecitabine and oxaliplatin for gastric cancer after D2 gastrectomy (CLASSIC): a phase 3 open-label, randomised controlled trial. Lancet. 2012;379 (9813):315-321. doi:10.1016/S0140-6736(11)61873-4

8. Noh SH, Park SR, Yang HK, et al. Adjuvant capecitabine plus oxaliplatin for gastric cancer after D2 gastrectomy (CLASSIC): 5-year follow-up of an open-label, randomised phase 3 trial. Lancet Oncol. 2014;15(12):1389-1396. doi:10.1016/S1470-2045(14)70473-5

9. Shitara K, Chin K, Yoshikawa T, et al. Phase II study of adjuvant chemotherapy of S-1 plus oxaliplatin for patients with stage III gastric cancer after D2 gastrectomy. Gastric Cancer. 2017;20 (1):175-181. doi:10.1007/s10120-015-0581-1

10. Wang G, Zhao J, Song Y, et al. Phase II study of adjuvant chemotherapy with $\mathrm{S} 1$ plus oxaliplatin for Chinese patients with gastric cancer. BMC Cancer. 2018;18(1):547. doi:10.1186/s12885-018-4480-9

11. Yang L, Yang Y, Qin Q, et al. Evaluation of the optimal dosage of S-1 in adjuvant SOX chemotherapy for gastric cancer. Oncol Lett. 2015;9 (3):1451-1457. doi:10.3892/ol.2014.2821

12. Sakuramoto S, Sasako M, Yamaguchi T, et al. Adjuvant chemotherapy for gastric cancer with S-1, an oral fluoropyrimidine. $N$ Engl $J$ Med. 2007;357(18):1810-1820. doi:10.1056/NEJMoa072252

13. Ter Veer E, Ngai LL, Valkenhoef GV, et al. Capecitabine, 5-fluorouracil and S-1 based regimens for previously untreated advanced oesophagogastric cancer: a network meta-analysis. Sci Rep. 2017;7(1):7142. doi:10.1038/s41598-017-07750-3

14. Miura K, Kinouchi M, Ishida K, et al. 5-fu metabolism in cancer and orally-administrable 5-fu drugs. Cancers (Basel). 2010;2 (3):1717-1730. doi:10.3390/cancers 2031717

15. Cheng X, Yu S, Wang Y, et al. The role of oxaliplatin in the adjuvant setting of different Lauren's type of gastric adenocarcinoma after D2 gastrectomy: a real-world study. Gastric Cancer. 2019;22 (3):587-597. doi:10.1007/s10120-018-0895-x

16. Ji J, Shen L, Li Z, et al. Perioperative chemotherapy of oxaliplatin combined with S-1 (SOX) versus postoperative chemotherapy of SOX or oxaliplatin with capecitabine (XELOX) in locally advanced gastric adenocarcinoma with D2 gastrectomy: a randomized phase III trial (RESOLVE trial). Ann Oncol. 2019;30(Suppl 5):v851-v934. doi:10.1093/annonc/mdz394.033

17. Nakamura Y, Yamanaka T, Chin K, et al. Survival outcomes of two phase 2 studies of adjuvant chemotherapy with S-1 plus oxaliplatin or capecitabine plus oxaliplatin for patients with gastric cancer after D2 gastrectomy. Ann Surg Oncol. 2019;26(2):465-472. doi:10.1245/ s10434-018-7063-8

18. Wang FH, Shen L, Li J, et al. The Chinese society of clinical oncology (CSCO): clinical guidelines for the diagnosis and treatment of gastric cancer. Cancer Commun (Lond). 2019;39(1):10. doi:10.1186/s40880-019-0349-9

19. Karavokyros I, Michalinos A. Favoring D2-lymphadenectomy in gastric cancer. Front Surg. 2018;5:42. doi:10.3389/fsurg.2018.00042

20. Kiyokawa T, Fukagawa T. Recent trends from the results of clinical trials on gastric cancer surgery. Cancer Commun (Lond). 2019;39 (1):11. doi:10.1186/s40880-019-0360-1 
21. Kim GM, Jeung HC, Rha SY, et al. A randomized phase II trial of S-1-oxaliplatin versus capecitabine-oxaliplatin in advanced gastric cancer. Eur J Cancer. 2012;48(4):518-526. doi:10.1016/j. ejca.2011.12.017

22. Ren DF, Zheng FC, Zhao JH, et al. Adjuvant chemotherapy with S-1 plus oxaliplatin improves survival of patients with gastric cancer after D2 gastrectomy: a multicenter propensity score-matched study. World J Clin Cases. 2018;6(10):373-383. doi:10.12998/wjcc.v6.i10.373

23. Yamada Y, Higuchi K, Nishikawa K, et al. Phase III study comparing oxaliplatin plus S-1 with cisplatin plus S-1 in chemotherapy-naive patients with advanced gastric cancer. Ann Oncol. 2015;26 (1):141-148. doi:10.1093/annonc/mdu472

24. Yoshida K, Kodera Y, Kochi M, et al. Addition of docetaxel to oral fluoropyrimidine improves efficacy in patients with stage iii gastric cancer: interim analysis of JACCRO GC-07, a randomized controlled trial. J Clin Oncol. 2019;37(15):1296-1304. doi:10.1200/ JCO.18.01138

25. Lee JL, Kang YK, Kang HJ, et al. A randomised multicentre phase II trial of capecitabine vs S-1 as first-line treatment in elderly patients with metastatic or recurrent unresectable gastric cancer. $\mathrm{Br} J$ Cancer. 2008;99(4):584-590. doi:10.1038/sj.bjc.6604536
26. Nishikawa K, Tsuburaya A, Yoshikawa T, et al. A randomised phase II trial of capecitabine plus cisplatin versus S-1 plus cisplatin as a first-line treatment for advanced gastric cancer: capecitabine plus cisplatin ascertainment versus S-1 plus cisplatin randomised PII trial (XParTS II). Eur J Cancer. 2018;101:220-228. doi:10.1016/j. ejca.2018.06.026

27. Rizzo A, Mollica V, Ricci AD, et al. Third- and later-line treatment in advanced or metastatic gastric cancer: a systematic review and meta-analysis. Future Oncol. 2020;16(2):4409-4418. doi:10.2217/ fon-2019-0429

28. Comets E, Ikeda K, Hoff P, Fumoleau P, Wanders J, Tanigawara Y. Comparison of the pharmacokinetics of S-1, an oral anticancer agent, in Western and Japanese patients. J Pharmacokinet Pharmacodyn. 2003;30(4):257-283. doi:10.1023/A:1026142601822

\section{Publish your work in this journal}

Cancer Management and Research is an international, peer-reviewed open access journal focusing on cancer research and the optimal use of preventative and integrated treatment interventions to achieve improved outcomes, enhanced survival and quality of life for the cancer patient.
The manuscript management system is completely online and includes a very quick and fair peer-review system, which is all easy to use. Visit http://www.dovepress.com/testimonials.php to read real quotes from published authors. 